\title{
Protein nanoparticle as a versatile drug delivery system in nanotechnology
}

\begin{abstract}
Protein nanoparticles are extensively investigated as versatile drug delivery systems, due to their nanoscale size, container-like shape, natural source, biocompatibility, and biodegradability. They provide three distinct interfaces: internal, external, and inter-subunit interfaces for modifications. Chemistry and genetic engineering provide powerful tools to functionalize protein nanoparticles. Through modifications to the three interfaces, protein nanoparticles can be functionalized with drug encapsulation, controlled drug release, controlled disassembly, and tumor targeting capabilities. This mini review introduced the fundamentals of protein nanoparticles as drug delivery systems, focused on modifications to the three different interfaces, and elucidated different strategies and mechanisms used in integrating multiple functionalities in protein nanoparticles.
\end{abstract}

Keywords: Drug delivery,Targeted Drug delivery, Protein nanoparticles, Capsid proteins, E2 nanoparticles, Drug encapsulation, Drug release, Tumor targeting particles
Volume 4 Issue I - 2016

\author{
Dongmei Ren \\ Department of Chemical Engineering and Materials Science, \\ University of California, USA
}

Correspondence: Dongmei Ren, Enzyme Technologies, BASF Enzymes LLC, 3550 John Hopkins Court, San Diego, California, 92I2I, USA, Tel I 858 43 I-8525 Email dmen 1222@gmail.com

Received: May 20, 2016 | Published: August I, 2016
Abbreviations:FDA, Food and Drug Administration; CCMV, Cowpea Chlorotic Mottle Virus; TMV, Tobacco Mosaic Virus; HCRSV, Hibiscus Chlorotic Ring Spot Virus; CMV, Cucumber Mosaic Virus; HSP, Heat Shock Proteins; HBV, Hepatitis B Virus; EGFR, Epidermal Growth Factor Receptor; PEG, Polyethylene Glycol

\section{Introduction}

Various therapeutic molecules and drugs have been developed to deal with all kinds of diseases. Drug discovery research identifies hundreds of new drugs each year to treat diverse diseases. However, some indigenous properties of drugs such as poor solubility, poor permeability, systematic toxicity, and short half lives limit their applications. Due to such challenges, U.S. Food and Drug Administration (FDA) only approved forty-five novel drugs in 2015 among the numerous drugs discovered. In order to overcome the limitations of drugs, drug delivery technology is developed to transport drug molecules more efficiently, effectively, and safely to the diseased areas. Drug delivery is especially important in cancer therapy. Chemotherapy is conventionally used to treat cancers; however, the strong therapeutic efficacy of cancer drugs can also damage healthy cells in humans, thus induce significant side effects in patients. Targeted drug delivery has been extensively studied to deliver cancer drugs specifically to tumors without negative impact on healthy organs, which can significantly decrease systematic toxicity and side effects compared to traditional chemotherapy. Therefore, drug delivery systems are very important in biomedical research, and combination of drug discovery and drug delivery can benefit the pharmaceutical industry.

An ideal drug delivery system incorporates drug encapsulation, controlled drug release, and specific targeting functionalities. Many materials have been designed as drug carriers, including protein nanoparticles, liposomes, dendrimers, hydrogels, carbon nanotubes, silica nanoparticles, etc. ${ }^{1-3}$ Among these materials, protein nanoparticles demonstrate several advantages over polymeric nanoparticles and inorganic materials. Protein nanoparticles are typically synthesized in microorganisms, which endow them with narrow and uniform size distribution due to the reliable protein synthesis pathway in microbes; protein nanoparticles are biodegradable, they can be readily degraded into non-toxic and biocompatible amino acids; protein nanoparticles are also apt to modifications by recombinant technology and chemistry, therefore different functionalities can be incorporated. Due to the reliable size, biodegradability, and ease of manipulation, protein nanoparticles have been widely investigated as versatile drug delivery systems.

\section{Discussion}

Nature has provided numerous candidates as drug delivery systems. One common category is viral protein capsids. All viruses consist of the capsid outside and the viral RNA inside. The capsid is composed of proteins, and serves as a shell to protect the internal viral RNA. The function of capsids in virus is one important property of drug carriers, to protect the encapsulated cargos from degradation. Inspired by this shell-core structure, researchers removed the viral nucleotides, and utilized the natural viral protein capsids as drug carriers. Researchers separated abundant capsids from their native viral RNA, and obtained the container-like caged protein particles. ${ }^{4}$ Various capsids investigated include Hepatitis B Virus (HBV), Cowpea Chlorotic Mottle Virus (CCMV), Tobacco Mosaic Virus (TMV), Cucumber Mosaic Virus (CMV), Hibiscus Chlorotic Ring Spot Virus (HCRSV), etc. ${ }^{4-5}$ Besides capsid proteins, there are also non-viral sourced caged proteins exploited as drug carriers, such as ferritin. ${ }^{6}$ Heat Shock Proteins (HSP). ${ }^{7}$ and enzymes. ${ }^{8-9}$ These protein nanoparticles are all assembled from individual subunits and form defined sizes and shapes. The size of protein cages is typically between $10 \mathrm{~nm}$ to 100 $\mathrm{nm}$, which is the desired size range for nanoparticle uptake by cells. ${ }^{9}$

Protein nanoparticles provide three interfaces to be redesigned for drug delivery purposes: the internal, external, and inter-subunit surfaces. The three interfaces can all be modified genetically or chemically to integrate multiple functionalities. The internal surface can be modified to encapsulate cargo molecules, such as imaging agents. ${ }^{10}$ drug molecules, and nucleotides. ${ }^{11}$ Controlled drug release feature can also be designed on the internal surface. Drug release can be triggered by various stimuli including temperature, $\mathrm{pH}$, light, etc. ${ }^{12}$ The external surface is generally used to integrate targeting functionalities. Targeting ligands or peptides ${ }^{11,13,14}$ can be attached to the external surfaces of protein nanoparticles. Through the specific and high affinity between targeting ligands/peptides and receptors over expressed by tumor cells, nanoparticles can bind to tumor 
cells specifically. The external surface can also be functionalized with immunological properties to induce immune responses and nanoparticles can serve as vaccines. ${ }^{15}$ The inter-subunit interactions can be modulated to control the assembly and disassembly of nanoparticles. Since protein nanoparticles are consisted of individual subunits, by modifications to subunits, the interactions between subunits can be modified and promote drug release. ${ }^{16}$

To encapsulate drug molecules and enable controlled drug release from nanoparticles, chemical conjugation approach is extensively investigated. Amino acid residues and other functional groups from protein nanoparticles provide active sites for drug conjugation; such active sites include cysteines,,${ }^{8,17}$ amines, ${ }^{18}$ carboxyl,,${ }^{19}$ tyrosines ,$^{20}$ phenol, guanidine, and imidazole. ${ }^{9,21}$ These active sites can be either native or introduced by mutagenesis. Hooker et al. ${ }^{20}$ isolated MS2 viral capsid particles by removing the RNA genome. They modified the native tyrosine residues in the interior of the capsid through diazonium-coupling reaction, for the attachment of olefin substrates. This strategy could be used to attach drug cargos or serve as nucleation sites. ${ }^{20}$ The internal surface of the protein shell of the Tobacco Mosaic Virus (TMV) was also modified. Schlick et al., ${ }^{18}$ exploited a carbodiimide coupling reaction and attached amines to glutamic acid side chains in the cavity of TMV particles for drug and gene delivery.18 Steinmetz et al., ${ }^{19}$ also functionalized the internal glutamic acid residues in TMV capsid and attached optical and MR contrast agents. ${ }^{22}$

In addition, acid-liable chemical bonds are widely used to trigger drug release, due to their $\mathrm{pH}$-responsive hydrolysis. These chemical bonds remain intact at neutral $\mathrm{pH}$, while at acidic environment, the chemical bonds can be hydrolyzed, and drugs attached through the linkage can be released. During endocytic uptake of nanoparticles by cells, nanoparticles will go through physiological $\mathrm{pH}$ change from extracellular neutral $\mathrm{pH} 7.4$ to intracellular acidic $\mathrm{pH}$, where $\mathrm{pH}$ could drop to $\mathrm{pH}<6.0 .{ }^{12}$ Hydrazone bond is often used in this strategy. Thiol group of cysteines can react with maleimide group and form a hydrazone linkage. Wang et al. ${ }^{13}$ introduced sixty cysteines to the internal surface of virus-like protein nanoscaffolds. They conjugated antitumor drug doxorubicin to the internal surface of the scaffolds through hydrazone linkage, and achieved $\mathrm{pH}$-dependent drug release. The protein-drug complex exhibited significant toxicity in cancer cells. ${ }^{8-10}$ Additionally, Huang et al. ${ }^{23}$ functionalized sericin protein with hydrazine, and doxorubicin was covalently conjugated to sericin through hydrazone bond. The sericin-doxorubicin conjugates selfassembled to nanoconjugates. The sericin nanoparticles encapsulating drugs showed significant drug release at $\mathrm{pH} 5.0$, and demonstrated antitumor efficacy. ${ }^{23}$

Besides chemical conjugation approach, other non-covalent interactions have also been used to encapsulate drug cargos in protein nanoparticles. Steinmetz et al. ${ }^{24}$ designed a straight forward protocol, and encapsulated antitumor drug phenanthriplatin to the interior of TMV nanoparticles through electrostatic gating. ${ }^{24}$ In order to encapsulate drugs into albumin particles, Yuan et al. ${ }^{25}$ exposed the hydrophobic domains of albumins to bind hydrophobic drugs, and then refolded into particles.$^{25}$ On the other hand, Wang et al. ${ }^{26}$ introduced hydrophobic amino acids and created a hydrophobic environment inside a protein nanoparticle to enhance its loading capacity for hydrophobic drugs. ${ }^{26}$ As summarized above, drug encapsulation can be through chemical conjugation or non-covalent interactions. They both have distinct advantages and limitations, therefore combination of different strategies can provide powerful tools for drug encapsulation.
Another area investigated in protein nanoparticles is the intersubunit interaction. Protein nanoparticles are assembled from a limited number of subunits. By adjusting the subunit-subunit interaction, the assembly behavior and architecture of nanoparticles can be controlled. ${ }^{16,27}$ One such example is a caged protein nanoparticle from the E2 component of pyruvate dehydrogenase complex. The E2 nanoparticle consists of sixty subunits, which self-assemble into a $\sim 26 \mathrm{~nm}$ particle ${ }^{28}$ Wang et al. ${ }^{29}$ genetically truncated part of the $\mathrm{N}$-terminal amino acid sequence of the $\mathrm{E} 2$ subunits to alter the intersubunit interaction, and synthesized a $\mathrm{pH}$-responsive nanoparticle. The particle remained intact at $\mathrm{pH} 7.4$, but dissociated at $\mathrm{pH} 5.0 .{ }^{16}$ This behavior can be used to trigger drug release. They also investigated the feasibility of utilizing histidines at the interfaces to control nanoparticle disassembauly. ${ }^{29}$ Lim et al. ${ }^{30}$ further identified the critical sites and introduced histidines at the inter and intra trimer interfaces of E2 nanoparticles to impart pH-responsive disassembly. ${ }^{30}$ To further understand the disassembly mechanism of E2 nanoparticles, they truncated the $\mathrm{C}$-terminus of the subunits and isolated the trimer intermediates. ${ }^{31}$ This discovery demonstrated the important role of inter-subunit interaction in protein particle self-assembly. The intersubunit interactions of ferritin A from E.coli were also investigated. Ferritin A is a 24-mer nanoparticle with 4-, 3- and 2-fold symmetric axes. Ohtomo et al..$^{32}$ investigated the interfaces at the three symmetric axes. They further truncated the C-terminus of one helix from the subunit, and disrupted the nanocage structure, indicating the C-terminus of subunits is critical in protein assembly. ${ }^{32}$ Discovery of Ohtomo et al. ${ }^{32}$ is consistent with Lim group's C-terminus truncation in E2 particles; even the two protein nanoparticles are from different classes and have distinct geometries and structures. In spite of different origins, protein nanoparticles are built from small subunits; the interactions between subunits can be redesigned and manipulated to impart diverse functionalities. Jian Zhang et al. ${ }^{33}$ reviewed more work on inter-subunit interfaces and subunit assembly in proteinbased assemblies. ${ }^{33}$

Exterior of protein nanoparticles is exploited in targeted drug delivery. Targeted drug delivery aims to direct drug carriers directly to tumor cells through targeting ligands. Targeting ligands are molecules that can recognize and specifically bind to receptors, which are over-expressed in tumor cells. Researchers have identified abundant targeting ligands, including small molecules such as folic acid/ folate. ${ }^{10,23,34}$ nucleic acid aptamers. ${ }^{35}$ antibodies, antibody fragments. ${ }^{36}$ and peptides. ${ }^{37}$ Huang et al. ${ }^{23}$ attached folate to sericin nanoparticles and demonstrated targeted cytotoxicity in $\mathrm{KB}$ cells over expressing folate receptors. ${ }^{23}$ Folic acid was also conjugated to soy protein nanoparticles encapsulating curcumin model drug. Cellular study revealed that the folic acid functionalized nanoparticles increased the uptake of nanoparticle in Caco-2 cells compared to non-targeting particles. ${ }^{34}$ Tong et al. ${ }^{35}$ utilized nucleic acid aptamers as targeting ligands. They attached nucleic acid aptamers of a 41-nucleotide sequence to the external surface of a MS2 viral capsid through oxidative coupling strategy. The resultant capsids specifically bind to Jurkat $\mathrm{T}$ cells expressing tyrosine kinase receptor. ${ }^{35}$ Antibodies and antibody fragments are also frequently used as targeting ligands due to their specific affinity to antigens. ${ }^{38,39}$ De Rose et al. ${ }^{38}$ functionalized caveolin-1 nanoparticles with monoclonal antibodies, and the specific binding of nanoparticles towards targeted immune cells was significantly increased. ${ }^{38}$ On the other hand, Lim et al. ${ }^{36}$ conjugated antibody fragments to the exterior of protein nanoparticles through a maleimide linker to target Epidermal Growth Factor Receptor (EGFR). The targeting nanoparticles can specifically bind to EGFRpositive cancer cells. ${ }^{36}$ 
Targeting peptide is another important targeting ligand used. Phage display technology allows the identification of numerous targeting peptides for different receptors. ${ }^{40,41}$ Guan et al. ${ }^{13}$ utilized genetic engineering approach and synthesized protein nanoparticles carrying enhanced green fluorescent protein and tat peptide, a cell-penetrating peptide. In vivo studies showed preferable accumulation of these targeting fluorescent nanoparticles in tumors.${ }^{13} \mathrm{Kim}$ et al. ${ }^{14}$ integrated different targeting peptides on the surface of ferritin nanoparticles. They displayed AP1 targeting peptides on the nanoparticles, which showed strong affinity to tumor cells over-expressing IL-4 receptors. Moreover, they fused RGD peptides and AP1 peptides simultaneously to the external surface of ferritin nanoparticles. The resultant nanoparticles are bispecific, and can target blood vessels and tumor cells respectively. ${ }^{14}$ Besides targeting capabilities, the exterior of protein nanoparticles can also be modified to induce immune responses, and nanoparticles can potentially be exploited as vaccine carriers. It has been reported that attachment of Polyethylene Glycol (PEG) to nanoparticles can help evade immune recognition. Molino et al. ${ }^{42}$ attached PEG molecules to the exterior of protein nanoparticles and reported decreased cellular uptake of nanoparticles. These PEGylated nanoparticles could also induce complement activation. ${ }^{42}$ To examine the possibility of using protein nanoparticles as vaccine carriers, Molino et al. ${ }^{15}$ conjugated $\mathrm{CpG}$ to the interior and gp100 epitope to the exterior of protein nanoparticles. Significant immune responses were observed in both in vitro and in vivostudies with these functionalized nanoparticles. ${ }^{15}$ The foregoing reviewed modifications to the exterior of protein nanoparticles could be achieved through either chemical conjugation strategy or recombinant strategy. Depending on the properties of the nanoparticles and the moieties to display, appropriate strategies need to be designed.

Besides the advantages mentioned above, protein nanoparticles also have limitations as drug delivery systems compared to inorganic and polymer based nanoparticles. Protein nanoparticles are typically synthesized from microorganisms. It is time consuming to produce and purify protein nanoparticles from microorganisms. In addition, some proteins require optimal storage conditions such as $\mathrm{pH}$ and temperature or other formulation conditions, due to stability and solubility issues. Moreover, it is challenging to introduce functionalities to protein nanoparticles. Protein nanoparticles exist in integral three dimensional structures. Modifications to protein nanoparticles may potentially disrupt the intact structure, result in disassembly, or change the properties of particles. Therefore it is tactical to choose appropriate sites within protein nanoparticles to introduce various moieties without disrupting the structure of particles. ${ }^{16,26,28}$ In order to design protein nanoparticles as drug delivery systems, these limitations need to be considered.

\section{Conclusion}

In summary, protein nanoparticles can be modified at the internal, external, and inter-subunit interfaces. These modifications could also be simultaneously integrated into one nanoparticle to impart multifunctionalties, such as drug loading, drug release, and tumor targeting functionalities. Chemical conjugation and recombinant strategies can be used to manipulate protein nanoparticles to achieve targeted delivery of diverse therapeutic molecules. Protein nanoparticle is a versatile platform for drug delivery.

\section{Acknowledgments}

None.

\section{Conflicts of interest}

None.

\section{References}

1. Cho K, Wang X, Nie S et al. Therapeutic Nanoparticles for Drug Delivery in Cancer. Clin Cancer Res. 2008;14(5):1310-1316.

2. Sood N, Bhardwaj A, Mehta S, Mehta A Stimuli-responsive hydrogels in drug delivery and tissue engineering. Drug Deliv. 2016;23(3):748-770.

3. Yang YN, Yu CZ Advances in silica based nanoparticles for targeted cancer therapy. Nanomed Nanotechnol Biol Med. 2016;12(2):317-332.

4. van Kan-Davelaar HE, van Hest JCM, Cornelissen JJ et al. Using viruses as nanomedicines. Br J Pharmacol. 2014;171(17):4001-4009.

5. Glasgow J, Tullman Ercek D (2014) Production and applications of engineered viral capsids. Appl Microbiol Biotechnol. 98(13): 5847-5858.

6. Truffi M, Fiandra L, Sorrentino L et al. Ferritin nanocages: A biological platform for drug delivery, imaging and theranostics in cancer. Pharmacol Res. 2016;107:57-65.

7. Flenniken ML, Willits DA, Brumfield S et al. The Small Heat Shock Protein Cage from Methanococcus jannaschii Is a Versatile Nanoscale Platform for Genetic and Chemical Modification. Nano Lett. 2003;3(11):1573-1576.

8. Ren D, Kratz F, Wang SW Protein Nanocapsules Containing Doxorubicin as a pH-Responsive Delivery System. Small. 2011;7(8):1051-1060.

9. Molino NM, Wang SW Caged protein nanoparticles for drug delivery. Curr Opin Biotechnol. 2014;28:75-82.

10. Ren D, Kratz F, Wang SW Engineered drug-protein nanoparticle complexes for folate receptor targeting. Biochem Eng J. 2014;89:33-41.

11. Elzoghby AO, Samy WM, Elgindy NA Protein-based nanocarriers as promising drug and gene delivery systems. $J$ Controlled Release. 2012;161(1):38-49.

12. Lu Y, Sun W, Gu Z Stimuli-responsive nanomaterials for therapeutic protein delivery. J Controlled Release. 2014;194:1-19.

13. Guan X, Li C, Wang D et al. A tumor-targeting protein nanoparticle based on Tat peptide and enhanced green fluorescent protein. RSC Adv 2016;6(12):9461-9464.

14. Kim S, Jeon JO, Jun E et al. Designing Peptide Bunches on Nanocage for Bispecific or Superaffinity Targeting. Biomacromolecules. 2016;17(3):1150-1159.

15. Molino NM, Neek M, Tucker JA et al. Viral-mimicking protein nanoparticle vaccine for eliciting anti-tumor responses. Biomaterials. 2016;86:83-91

16. Dalmau M, Lim S Wang SW Design of a pH-Dependent Molecular Switch in a Caged Protein Platform. Nano Lett. 2009;9(1):160-166.

17. Tian Y, Gao S, Wu M et al. Tobacco Mosaic Virus-Based 1D NanorodDrug Carrier via the Integrin-Mediated Endocytosis Pathway. ACS Appl Mater Interfaces. 2016;8(17):10800-10807.

18. Schlick TL, Ding Z, Kovacs EW, Francis MB Dual-Surface Modification of the Tobacco Mosaic Virus. J Am Chem Soc. 2005;127(11):3718-3723.

19. Aljabali AAA, Shukla S, Lomonossoff GP et al. CPMV-DOX Delivers. Mol Pharmaceutics 10(1): 3-10.

20. Hooker JM, Kovacs EW, Francis MB Interior Surface Modification of Bacteriophage MS2. J Am Chem Soc. 2004;126(12):3718-3719.

21. Lohcharoenkal W, Wang L, Chen YC, Rojanasakul Y Protein nanoparticles as drug delivery carriers for cancer therapy. BioMed Res Int p.12. 2014

22. Bruckman MA, Jiang K, Simpson EJ et al. Dual-Modal Magnetic Resonance and Fluorescence Imaging of Atherosclerotic Plaques in Vivo Using VCAM-1 Targeted Tobacco Mosaic Virus. Nano Lett. 2014;14(3): 1551-1558 
23. Huang L, Tao K, Liu J et al. Design and Fabrication of Multifunctional Sericin Nanoparticles for Tumor Targeting and $\mathrm{pH}-$ Responsive Subcellular Delivery of Cancer Chemotherapy Drugs. ACS Appl Mater Interfaces. 2014;8(10):6577-6585.

24. Czapar AE, Zheng YR, Riddell IA et al. Tobacco Mosaic Virus Delivery of Phenanthriplatin for Cancer therapy. ACS Nano. 2016;10(4):4119-4126.

25. Yuan A, Wu J, Song $\mathrm{C}$ et al. A novel self-assembly albumin nanocarrier for reducing doxorubicin-mediated cardiotoxicity. J Pharm Sci. 2013;102(5):1626-1635.

26. Wang SW, Ren D, Dalmau M et al. Biomimetic Design of Protein Nanomaterials for Hydrophobic Molecular Transport. Adv Funct Mater. 2012;22(15):3170-3180.

27. Uchida M, Klem MT, Allen M et al. Biological Containers: Protein Cages as Multifunctional Nanoplatforms. Adv Mater. 2007;19(8):1025-1042.

28. Dalmau M, Lim S, Chen HC et al. Thermostability and molecular encapsulation within an engineered caged protein scaffold. Biotechnol Bioeng. 2008;101(4):654-664.

29. Dalmau M, Lim S, Wang SW pH-Triggered Disassembly in a Caged Protein Complex. Biomacromolecules. 2009;10(12):3199-3206.

30. Peng T, Lim S Trimer-Based Design of $\mathrm{pH}-$ Responsive Protein Cage Results in Soluble Disassembled Structures. Biomacromolecules. 2011;12(9):3131-3138.

31. Peng $\mathrm{T}$, Lee $\mathrm{H}$, Lim $\mathrm{S}$ Isolating a Trimer Intermediate in the SelfAssembly of E2 Protein Cage. Biomacromolecules. 2012;13(3):6 99-705.

32. Ohtomo H, Ohtomo M, Sato D et al. A Physicochemical and Mutational Analysis of Intersubunit Interactions of Escherichia coli Ferritin A. Biochemistry. 2015;54(40):6243-6251.
33. Zhang J, Zheng F, Grigoryan G Design and designability of proteinbased assemblies. Curr Opin Struct Biol. 2014;27:79-86.

34. Teng Z, Luo Y, Wang $\mathrm{T}$ et al. Development and Application of Nanoparticles Synthesized with Folic Acid Conjugated Soy Protein. $J$ Agric Food Chem. 2013;61(10):2556-2564.

35. Tong GJ, Hsiao SC, Carrico ZM et al. Viral Capsid DNA Aptamer Conjugates as Multivalent Cell-Targeting Vehicles. J Am Chem Soc. 2009;131(31):11174-11178.

36. Buecheler JW, Howard CB, de Bakker CJ et al. Development of a protein nanoparticle platform for targeting EGFR expressing cancer cells. J Chem Technol Biotechnol. 2015;90(7):1230-1236.

37. Raucher D, Ryu JS Cell-penetrating peptides: strategies for anticancer treatment. Trends Mol Med. 21(9):560-570.

38. Glass JJ, Yuen D, Rae J, et al. Human immune cell targeting of protein nanoparticles - caveospheres. Nanoscale. 2016;8(15):8255-8265.

39. Nelson ALAntibody fragments: Hope and hype. MAbs. 2010;2(1):77-83.

40. Svensen N, Walton JGA, Bradley M Peptides for cell-selective drug delivery. Trends Pharmacol Sci. 2012;33(4):186-192.

41. Ruoslahti E Peptides as Targeting Elements and Tissue Penetration Devices for Nanoparticles. Adv Mater. 2012;24(28):3747-3756.

42. Molino NM, Bilotkach K, Fraser DA et al. Complement Activation and Cell Uptake Responses Toward Polymer-Functionalized Protein Nanocapsules. Biomacromolecules. 2012;13(4):974-981. 